\title{
Divisibility criteria for class numbers of imaginary quadratic fields
}

\author{
by
}

Paul Jenkins (Los Angeles, CA) and Ken Ono (Madison, WI)

1. Introduction and statement of results. Throughout, let $d \equiv 0,3$ $(\bmod 4)$ be a positive integer, and let $\mathcal{Q}_{d}$ denote the set of positive definite integral binary quadratic forms $Q(x, y)=a x^{2}+b x y+c y^{2}=[a, b, c]$ with discriminant $-d=b^{2}-4 a c$ (including imprimitive forms if there are any). The group $\Gamma:=\mathrm{PSL}_{2}(\mathbb{Z})$ acts on $\mathcal{Q}_{d}$ with finitely many orbits, and if $\omega_{Q}$ is defined by

$$
\omega_{Q}= \begin{cases}2 & \text { if } Q \sim_{\Gamma}[a, 0, a] \\ 3 & \text { if } Q \sim_{\Gamma}[a, a, a], \\ 1 & \text { otherwise }\end{cases}
$$

then the Hurwitz-Kronecker class number $H(-d)$ is given by

$$
H(d)=\sum_{Q \in \mathcal{Q}_{d} / \Gamma} \frac{1}{\omega_{Q}} .
$$

If $-d<-4$ is a fundamental discriminant, then $H(-d)$ is the class number of the ring of integers of the imaginary quadratic field $\mathbb{Q}(\sqrt{-d})$.

Recently, Guerzhoy has obtained some interesting expressions for

$$
\left(1-\left(\frac{-d}{p}\right)\right) H(-d)
$$

as $p$-adic limits of traces of singular moduli. To make this precise, we first recall some notation. For positive definite binary quadratic forms $Q$, let $\alpha_{Q}$ be the unique root of $Q(x, 1)=0$ in the upper half of the complex plane. If $j(z)$ is the usual $\mathrm{SL}_{2}(\mathbb{Z})$ modular function

$$
j(z)=\frac{E_{4}(z)^{3}}{\Delta(z)}=q^{-1}+744+196884 q+\cdots,
$$

2000 Mathematics Subject Classification: 11R29, 11F37.

Key words and phrases: class numbers, singular moduli.

The authors thank the National Science Foundation for their generous support. The second author is grateful for the support of a Packard and a Romnes Fellowship. 
where $q=e^{2 \pi i z}$, then define integers $\operatorname{Tr}(d)$ by

$$
\operatorname{Tr}(d)=\sum_{Q \in \mathcal{Q}_{d} / \Gamma} \frac{j\left(\alpha_{Q}\right)-744}{\omega_{Q}} .
$$

The algebraic integers $j\left(\alpha_{Q}\right)$ are known as singular moduli. Guerzhoy proved (see Corollary 2.4(a) of [5]) that if $p \in\{3,5,7,13\}$ and $-d<-4$ is a fundamental discriminant, then one has the $p$-adic limit formula

$$
\left(1-\left(\frac{-d}{p}\right)\right) \cdot H(-d)=\frac{p-1}{24} \lim _{n \rightarrow \infty} \operatorname{Tr}\left(p^{2 n} d\right) .
$$

If $\left(\frac{-d}{p}\right)=1$, then this result simply implies that $\operatorname{Tr}\left(p^{2 n} d\right) \rightarrow 0 p$-adically as $n$ tends to infinity. Thanks to work of Boylan, Edixhoven and the first author (see $[2,4,6])$, it turns out that more is true. In particular, if $p$ is any prime and $\left(\frac{-d}{p}\right)=1$, then

$$
\operatorname{Tr}\left(p^{2 n} d\right) \equiv 0\left(\bmod p^{n}\right) .
$$

In earlier work [3], Bruinier and the second author obtained certain $p$ adic expansions for $H(-d)$ in terms of the Borcherds exponents of certain modular functions with Heegner divisor. In his paper [5], Guerzhoy asks whether there is a connection between $(1.3)$ and these results when $\left(\frac{-d}{p}\right) \neq 1$. In this note we show that this is indeed the case by establishing the following congruences.

Theorem 1.1. Suppose that $-d<-4$ is a fundamental discriminant and that $n$ is a positive integer. If $p \in\{2,3\}$ and $\left(\frac{-d}{p}\right)=-1$, or $p \in\{5,7,13\}$ and $\left(\frac{-d}{p}\right) \neq 1$, then

$$
\frac{24}{p-1} \cdot\left(1-\left(\frac{-d}{p}\right)\right) \cdot H(-d) \equiv \operatorname{Tr}\left(p^{2 n} d\right)\left(\bmod p^{n}\right) .
$$

In particular, under these hypotheses $p^{n}$ divides $\frac{24}{p-1}\left(1-\left(\frac{-d}{p}\right)\right) \cdot H(-d)$ if and only if $p^{n}$ divides $\operatorname{Tr}\left(p^{2 n} d\right)$.

Three remarks. 1) Theorem 1.1 includes $p=2$. For simplicity, Guerzhoy chose to work with odd primes $p$, and this explains the omission of $p=2$ in (1.3).

2) Despite the uniformity of (1.4), it turns out that the restriction on $p$ in Theorem 1.1 is required. For example, if $p=11, n=1$ and $-d=-15$, then $\left(\frac{-15}{11}\right)=-1, H(-15)=2$, and we have

$\operatorname{Tr}\left(11^{2} \cdot 15\right)$

$=-13374447806956269126908865521582974841084501554961922745794$

$$
\equiv 7 \not \equiv \frac{48}{10} \cdot H(-15)(\bmod 11) \text {. }
$$


3) There are generalizations of Theorem 1.1 which hold for primes $p \notin$ $\{2,3,5,7,13\}$. For example, one may employ Serre's theory [7] of $p$-adic modular forms to derive more precise versions of Corollary 2.4(b) of [5].

2. The proof of Theorem 1.1. The proof of Theorem 1.1 follows by combining earlier work of Bruinier and the second author with results of Zagier and a combinatorial formula used earlier by the first author. We recall some necessary notation.

Let $M_{\lambda+1 / 2}^{!}$be the space of weight $\lambda+1 / 2$ weakly holomorphic modular forms on $\Gamma_{0}(4)$ with Fourier expansion

$$
f(z)=\sum_{(-1)^{\lambda} n \equiv 0,1(\bmod 4)} a(n) q^{n} .
$$

For $0 \leq d \equiv 0,3(\bmod 4)$, we let $f_{d}(z)$ be the unique form in $M_{1 / 2}^{!}$with expansion

$$
f_{d}(z)=q^{-d}+\sum_{0<D \equiv 0,1(\bmod 4)} A(D, d) q^{D} .
$$

The coefficients $A(D, d)$ of the $f_{d}$ are integers. For completeness, we set $A(M, N)=0$ if $M$ or $N$ is not an integer. These modular forms are described in detail in [8].

For fundamental discriminants $-d<-4$, Borcherds' theory on the infinite product expansion of modular forms with Heegner divisor [1] implies that

$$
q^{-H(-d)} \prod_{n=1}^{\infty}\left(1-q^{n}\right)^{A\left(n^{2}, d\right)}
$$

is a weight zero modular function on $\mathrm{SL}_{2}(\mathbb{Z})$ whose divisor consists of a pole of order $H(-d)$ at infinity and a simple zero at each Heegner point of discriminant $-d$. Using this factorization, Bruinier and the second author proved the following theorem.

Theorem 2.1 ([3, Corollary 3]). Let $-d<-4$ be a fundamental discriminant. If $p \in\{2,3\}$ and $\left(\frac{-d}{p}\right)=-1$, or $p \in\{5,7,13\}$ and $\left(\frac{-d}{p}\right) \neq 1$, then as $p$-adic numbers we have

$$
H(-d)=\frac{p-1}{24} \sum_{k=0}^{\infty} p^{k} A\left(p^{2 k}, d\right) .
$$

REMARK. The case when $p=13$ is not proven in [3]. However, thanks to the remark preceding Theorem 8 of [7] on 13-adic modular forms with weight congruent to $2(\bmod 12)$, and Theorem 2 of [3], the proof of [3, Corollary 3 ] still applies mutatis mutandis. 
Zagier identified traces of singular moduli with the coefficients $A(D, d)$ as follows.

Theorem 2.2 ([8, Corollary to Theorem 3$])$. For all positive integers $d \equiv 0,3(\bmod 4)$,

$$
\operatorname{Tr}(d)=A(1, d) .
$$

Combining Zagier's duality ([8, Theorem 4]) between coefficients of modular forms in $M_{1 / 2}^{!}$and in $M_{3 / 2}^{!}$with the action of the Hecke operators on these spaces, the first author proved the following combinatorial formula.

Lemma 2.3 ([6, Theorem 1.1]). If $p$ is a prime and $d, D, n$ are positive integers such that $-d, D \equiv 0,1(\bmod 4)$, then

$$
\begin{aligned}
A\left(D, p^{2 n} d\right)= & p^{n} A\left(p^{2 n} D, d\right) \\
& +\sum_{k=0}^{n-1}\left(\frac{D}{p}\right)^{n-k-1}\left(A\left(\frac{D}{p^{2}}, p^{2 k} d\right)-p^{k+1} A\left(p^{2 k} D, \frac{d}{p^{2}}\right)\right) \\
& +\sum_{k=0}^{n-1}\left(\frac{D}{p}\right)^{n-k-1}\left(\left(\left(\frac{D}{p}\right)-\left(\frac{-d}{p}\right)\right) p^{k} A\left(p^{2 k} D, d\right)\right) .
\end{aligned}
$$

REMARK. This result is stated for odd $p$ in [6], but the proof holds for $p=2$ as well.

Proof of Theorem 1.1. Under the given hypotheses, Theorem 2.1 implies that

$$
\frac{24}{p-1} \cdot H(-d) \equiv \sum_{k=0}^{n-1} p^{k} A\left(p^{2 k}, d\right)\left(\bmod p^{n}\right) .
$$

By letting $D=1$ in Lemma 2.3, for these $d$ and $p$ we find that

$$
\left(1-\left(\frac{-d}{p}\right)\right) \sum_{k=0}^{n-1} p^{k} A\left(p^{2 k}, d\right)=A\left(1, p^{2 n} d\right)-p^{n} A\left(p^{2 n}, d\right) .
$$

Inserting this expression for the sum into (2.2), we conclude that

$$
\frac{24}{p-1} \cdot\left(1-\left(\frac{-d}{p}\right)\right) \cdot H(-d) \equiv A\left(1, p^{2 n} d\right)\left(\bmod p^{n}\right),
$$

which by Zagier's theorem is $\operatorname{Tr}\left(p^{2 n} d\right)$.

\section{References}

[1] R. E. Borcherds, Automorphic forms on $O_{s+2,2}(\mathbb{R})$ and infinite products, Invent. Math. 120 (1995), 161-213.

[2] M. Boylan, 2-adic properties of Hecke traces of singular moduli, Math. Res. Lett. 12 (2005), 593-609. 
[3] J. H. Bruinier and K. Ono, The arithmetic of Borcherds' exponents, Math. Ann. 327 (2003), 293-303.

[4] B. Edixhoven, On the p-adic geometry of traces of singular moduli, Int. J. Number Theory 1 (2005), 495-497.

[5] P. Guerzhoy, The Borcherds-Zagier isomorphism and a p-adic version of the KohnenShimura map, Int. Math. Res. Not. 2005, no. 13, 799-814.

[6] P. Jenkins, $p$-adic properties for traces of singular moduli, Int. J. Number Theory 1 (2005), 103-107.

[7] J.-P. Serre, Formes modulaires et fonctions zêta p-adiques, in: Modular Functions of One Variable, III (Antwerp, 1972), Lecture Notes in Math. 350, Springer, Berlin, 1973, 191-268.

[8] D. Zagier, Traces of singular moduli, in: Motives, Polylogarithms and Hodge Theory, Part I (Irvine, CA, 1998), Int. Press Lect. Ser. 3, Int. Press, Somerville, MA, 2002, 211-244.

Mathematics Department

UCLA

Los Angeles, CA 90095-1555, U.S.A.

E-mail: jenkins@math.ucla.edu
Department of Mathematics University of Wisconsin Madison, WI 53706, U.S.A. E-mail: ono@math.wisc.edu

Received on 20.3.2006

and in revised form on 5.9.2006 